\title{
X-ray Fluorescence Mapping of Transition Metals in Human KB Cells Undergoing Staurosporine-Induced Apoptosis
}

\author{
Jesse Ward*, Christopher Kelly**, Stefan Vogt*, Mark Banaszak Holl***, James Penner-Hahn*** \\ * Advanced Photon Source, Argonne National Laboratory, Argonne, IL, 60439 \\ ** School of Applied and Engineering Physics, Cornell University, Ithaca, NY, 14853 \\ *** Department of Chemistry, University of Michigan, Ann Arbor, MI, 48109
}

Apoptosis, or "programmed cell death", is the process by which multicellular organisms remove unwanted or damaged cells [1]. This process is essential for normal body plan development, and the failure of cells to respond to apoptotic stimuli is a risk factor for the development of cancer. Thus, it is desirable to understand what factors control the onset and progression of apoptosis. Over the past 30 years, many of these factors have been elucidated, including the roles of intracellular calcium, cysteine-aspartic acid proteases (caspases), and the Ras and Bcl-2 family proteins. However, the role, if any, that transition metal ions play in this process is less clear.

While transition metals such as iron, copper, and zinc comprise less than $0.01 \%$ of the human body by mass, life as we know it would not be possible without them [2]. Transition metal ions form complexes with biological macromolecules and are crucial for carrying out a variety of functions essential for life, including catalysis, electron transfer, structure stabilization, and oxygen storage and transport. Because of their versatility and ubiquity, these complexes are also expected to play a role in the regulation of apoptosis.

Of the transition metals, the connection between zinc and apoptosis has received the most attention, since both zinc deficiency and zinc excess prime cells for apoptosis [3]. High zinc levels have been found to inhibit cytochrome $\mathrm{c}$ release from the mitochondria, a crucial step in certain apoptotic pathways, as determined through the use of zinc-sensitive fluorophores [4]. However, it is unknown if this phenomenon occurs at physiological zinc levels. A similar study employing zinc-sensitive fluorophores under physiological conditions would face potential complications due to the fact that the mitochondria are involved in intracellular calcium storage, and that zinc-sensitive fluorophores sometimes experience cross-talk with other metal ions.

In order to address these issues, we employed hard X-ray fluorescence imaging of human KB cells undergoing staurosporine-induced apoptosis at the Advanced Photon Source at Argonne. This technique allows simultaneous, quantitative, and metal-specific determination of total metal levels and distributions on a single-cell level. X-ray fluorescence mapping reveals cytosolic redistribution of zinc and copper in the early stages after exposure to an apoptotic stimulus, which may indicate the presence of crucial metal-binding sites.

References

1. Elmore, S., Apoptosis: a review of programmed cell death. Toxicologic pathology, 2007. 35(4): p. 495. 
2. Kaim, W. and B. Schwederski, Bioinorganic Chemistry: Inorganic Elements in the Chemistry of Life: An Introduction and Guide. 1994, San Francisco, CA: John Wiley \& Sons.

3. Truong-Tran, A.Q., et al., The role of zinc in caspase activation and apoptotic cell death. Biometals, 2001. 14(3/4): p. 315.

4. Zamzami, N., et al., Mitochondrial control of nuclear apoptosis. J. Exp. Med., 1996. 183(4): p. $1533-1544$.
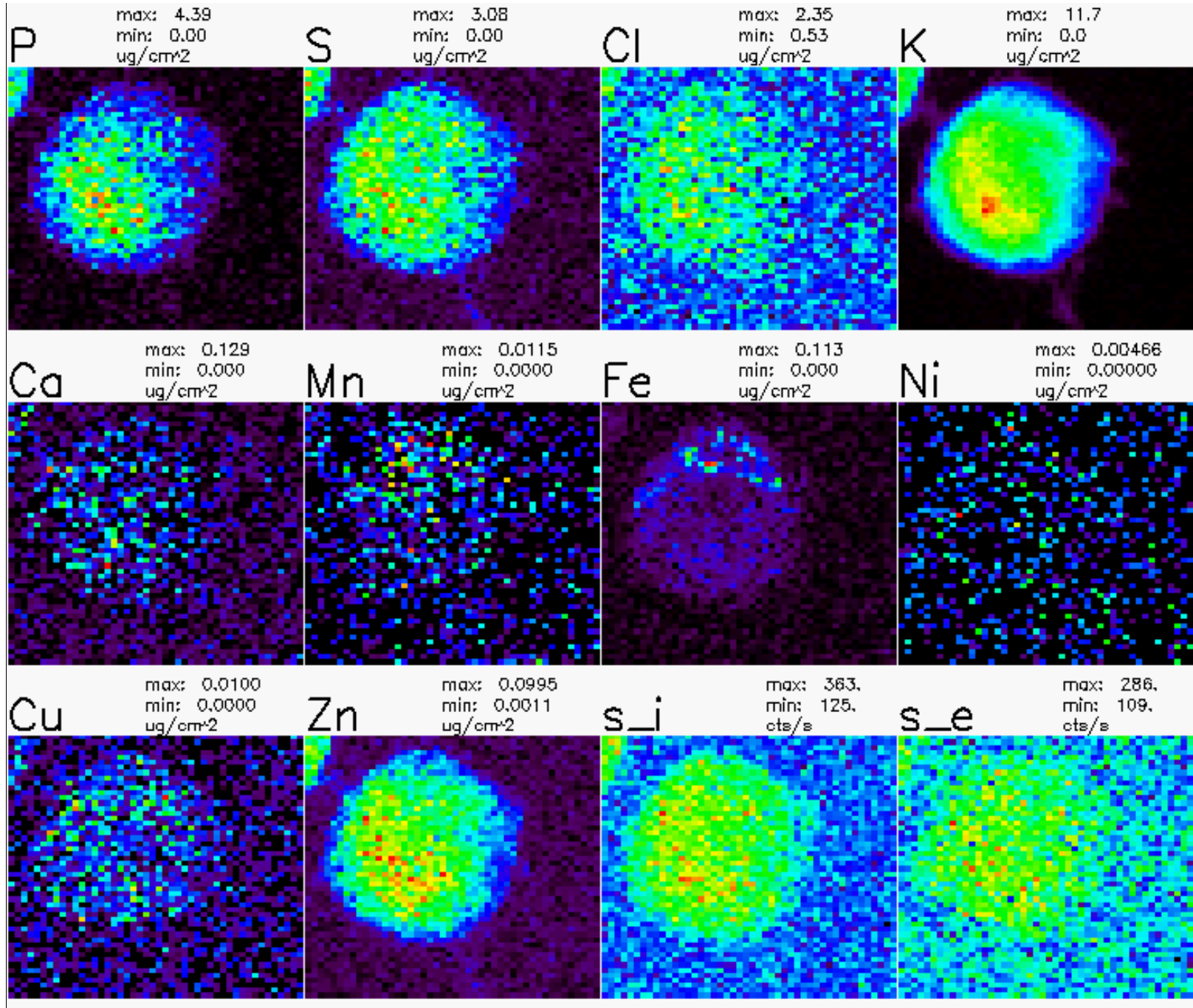

$\max : 286$
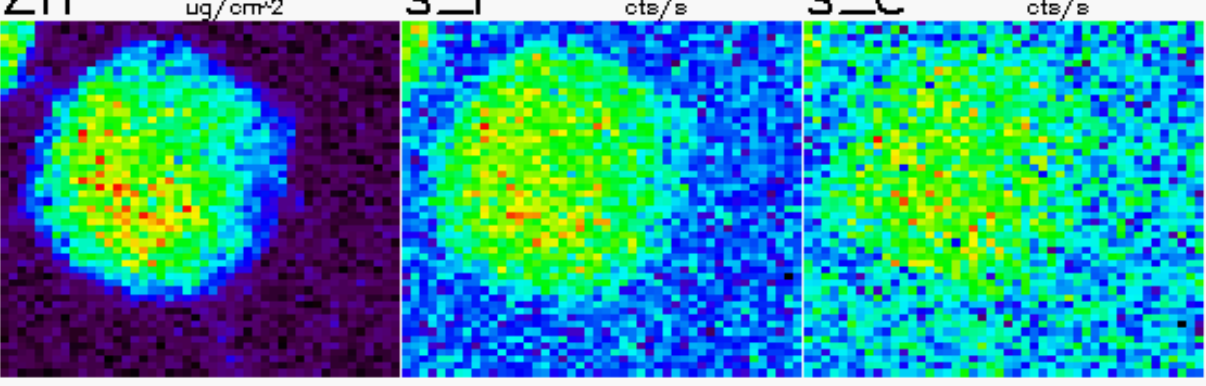

2 uा

FIG. Elemental maps within a single human KB cell. 